\title{
Transferable, transparent and functional polymer@graphene 2D objects
}

\author{
Tingting Gao ${ }^{1,2,3,6}$, Sze-Wing Ng${ }^{1,6}$, Xuqing Liu ${ }^{1}$, Liyong Niu ${ }^{1}$, Zhuang $\mathrm{Xie}^{1}$, Ruisheng Guo ${ }^{1,2,3}$, \\ Chaojian Chen ${ }^{1}$, Xuechang Zhou ${ }^{1}$, Jun $\mathrm{Ma}^{4}$, Wei Jin ${ }^{4}$, Ying-San Chui ${ }^{5}$, Wenjun Zhang ${ }^{5}$, \\ Feng Zhou ${ }^{2}$ and Zijian Zheng ${ }^{1}$
}

Compared with inorganic two-dimensional (2D) materials, such as graphene and transition metal dichalcogenides, organic 2D materials are believed to possess more interesting chemical and biological properties for certain applications, such as separation membranes, smart surfaces, sensors, catalysis and drug delivery. However, the study of organic 2D materials is largely hindered because of the lack of effective methods to produce them. This paper presents a new type of organic 2D material, namely 'polymer@graphene 2D objects', that can be synthesized via a simple and scalable chemistry.

Polymer@graphene 2D objects are made of functional polymer brushes that tether one end of the polymer chain on the surface of graphene sheets via non-covalent $\pi-\pi$ stacking interactions. These materials are transparent, freestanding, lightweight, flexible, transferable to various substrates with good stability and are patternable into different structures. Their functionality can be tailored by changing the polymer brushes that are immobilized. In this paper, we demonstrate the applications of these 2D objects in the smart control of surface wettability and DNA biosensors.

NPG Asia Materials (2014) 6, e130; doi:10.1038/am.2014.79; published online 26 September 2014

\section{INTRODUCTION}

Two-dimensional (2D) inorganic materials including graphene $e^{1-3}$ and various transition metal dichalcogenides ${ }^{4-6}$ have received tremendous attention in the past decade because of their unique properties and wide applications in optics and electronics. These remarkable phenomena have recently attracted research interest in $2 \mathrm{D}$ organic materials, including $2 \mathrm{D}$ polymers ${ }^{7-10}$ and quasi-2D polymers. ${ }^{11-15}$ The focus has mainly been on their chemical and biological properties and their applications as separation membranes, smart surfaces and sensors, and for catalysis and drug delivery. However, the 2D polymers reported to date are only limited to very specific building blocks and microscale sizes because of the lack of robust methods to produce them. On the other hand, the preparation of quasi-2D polymers either requires the undesirable crosslinking of the materials, using expensive and opaque novel metal supports, or high-energy electron beams. ${ }^{16,17}$ Therefore, the investigation of the fundamental properties as well as practical applications of these organic 2D materials have remained largely hindered.

In this paper, we report the development of a new type of functional organic 2D material, namely 'polymer@graphene 2D objects', which are prepared in a cost-effective and high-throughput manner.Polymer@graphene 2D objects are made of functional polymer brushes that tether one end of the polymer chain on the surface of graphene sheets via non-covalent $\pi-\pi$ stacking interactions (as illustrated in Scheme 1). Graphene acts as a mechanical support in this $2 \mathrm{D}$ object because of its atomic thickness, ultra flexibility, superb optical transparency ( $>97 \%$ in visible and infrared range), mechanical durability (Young's modulus: $\sim 1 \mathrm{TPa}$; tensile strength: $\sim 100$ GPa), stability in air and moisture below $300^{\circ} \mathrm{C} .{ }^{3,18-21}$ Meanwhile, polymer brushes tethered on the graphene surface provide desirable chemical and biological functionalities. Importantly, non-covalent $\pi$ $\pi$ stacking interactions are selected to immobilize functional polymer brushes because these interactions do not require a harsh reaction environment but offer remarkable stability for tethering the polymer chains on the graphene surface even using good solvents. Therefore, polymer@graphene 2D objects possess several unique characteristics: (i) These materials are mechanically freestanding, optically transparent and solvent-stable 2D ultrathin materials. (ii) The lateral size of the $2 \mathrm{D}$ objects can be readily scaled to be as large as the size of the graphene support. We demonstrate the preparation of a 3 -in sample in this paper. Additionally, these large-sized 2D objects can be patterned into different structures or smaller pieces using conventional lithography and soft lithography. (iii) A wide variety of chemical and biological functionalities can be achieved on these $2 \mathrm{D}$

\footnotetext{
${ }^{1}$ Nanotechnology Center, Institute of Textiles and Clothing, The Hong Kong Polytechnic University, Hong Kong, China; ${ }^{2}$ State Key Laboratory of Solid Lubrication, Lanzhou Institute of Chemical Physics, Chinese Academy of Sciences, Lanzhou, China; ${ }^{3}$ Graduate School of Chinese Academy of Sciences, Beijing, China; ${ }^{4}$ Department of Electrical Engineering, The Hong Kong Polytechnic University, Hong Kong, China and ${ }^{5}$ Center of Super-Diamond and Advanced Films (COSDAF), Department of Physics and Materials Science, City University of Hong Kong, Hong Kong, China

6These authors contributed equally to this work.

Correspondence: Professor Z Zheng or Professor F Zhou, Institute of Textiles and Clothing, The Hong Kong Polytechnic University, Hung Hom, Kowloon, Hong Kong. E-mail: tczzheng@polyu.edu.hk or zhouf@licp.cas.cn
}

Received 22 May 2014; revised 24 July 2014; accepted 24 July 2014 


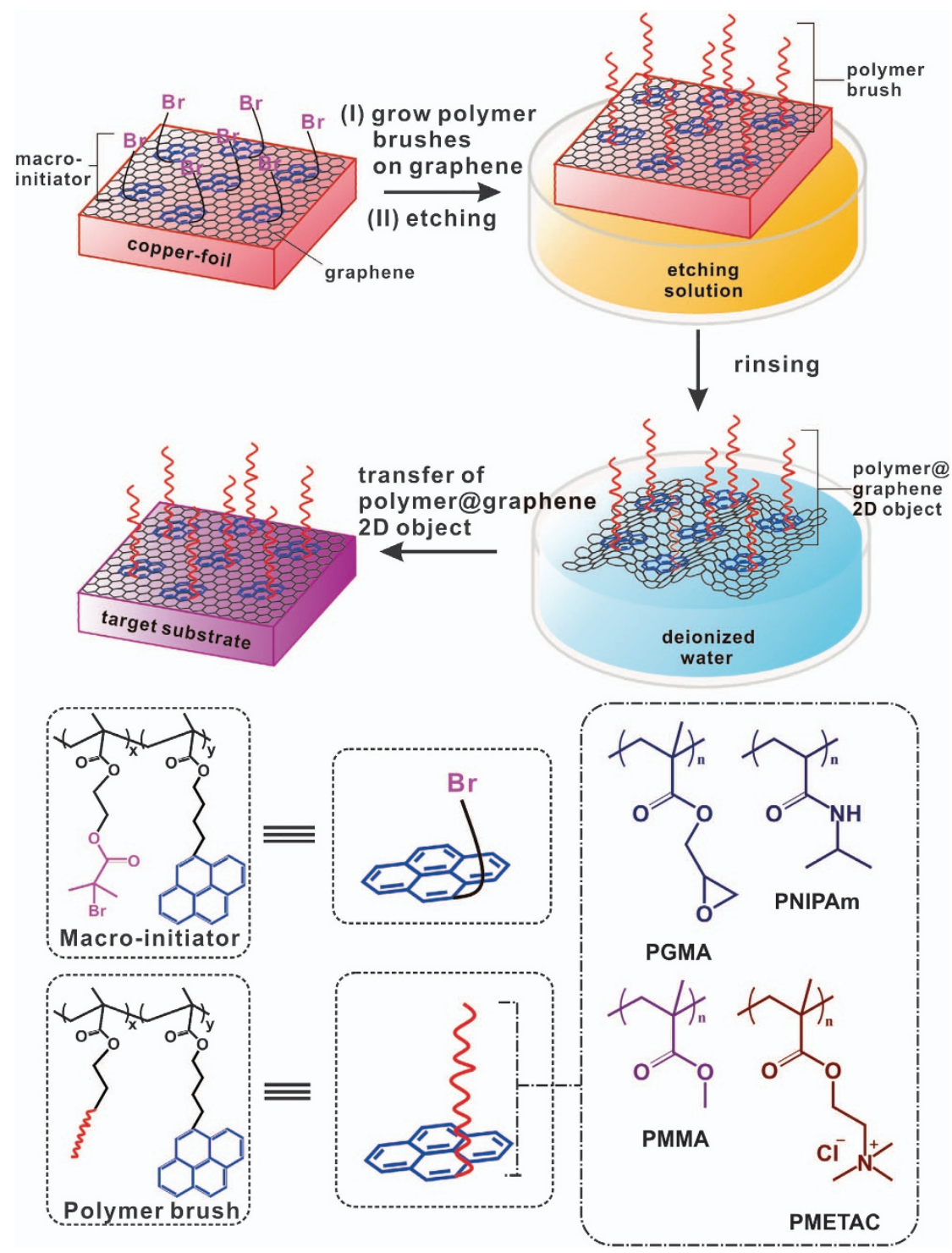

Scheme 1 Schematic illustration of the procedures for the fabrication and transfer of polymer@graphene 2D objects. The drawing is not to scale.

objects by simply synthesizing different polymer brushes. (iv) Polymer@graphene 2D objects can be readily transferred onto different substrates, even flexible ones, with excellent adhesion on the substrates. Importantly, this approach is an effective directtransfer method of rendering functionality to substrates that are otherwise difficult to modify using traditional wet chemistry. To demonstrate the uniqueness of polymer@graphene 2D objects, we present four types of homogeneous and patterned polymer@graphene 2D objects with excellent optical transparency and solvent stability that can be readily transferred onto different substrates for use as responsible surfaces or functional surfaces for immobilizing DNA oligonucleotide arrays.

\section{EXPERIMENTAL PROCEDURES}

\section{Materials}

The chemical vapor deposition (CVD)-grown graphene sheets on $\mathrm{Cu}$ foil were supplied by Apex Graphene Technology Co., Ltd. (Wanchai, Hong Kong) $\mathrm{FeCl}_{3}$, methyl methacrylate (MMA), (2-(methylacryloyloxyl)ethyl) trimethylammonium chloride (METAC), N-isopropyl acrylamide (NIPAm), glycidyl methacrylate (GMA) and pentamethyldiethylenetriamine were purchased from Sigma-Aldrich (Shanghai, PR China). The poly(ethylene terephthalate) (PET) films were supplied by the DuPont Company. DNA oligonucleotides were purchased from Takara (Dalian, China). The target sequence was $5^{\prime}-\mathrm{NH}_{2}$ $\left(\mathrm{CH}_{2}\right)_{6}$-CAT GAT TGA ACC ATC CAC CA-TET-3', and the probe sequence was $5^{\prime}$-TAMRA-TGG TGG ATG GTT CAA TCA TG-3'.

\section{Macro-initiator assembly}

The macro-initiator was synthesized according to a previous report. ${ }^{21}$ The Cu-foil-supported CVD graphene film was immersed in a $2 \mathrm{mg} \mathrm{ml}^{-1}$ macroinitiator solution (dimethylformamide (DMF)) for $24 \mathrm{~h}$ in the dark at room temperature. The film was rinsed with copious DMF and acetone to remove the physically adsorbed macro-initiator and then dried under $\mathrm{N}_{2}$ flow to prepare the macro-initiator-modified CVD-grown graphene film.

Surface-initiated atom transfer radical polymerization (SI-ATRP) SI-ATRP was performed in Schlenk tubes. The polymerization recipes for monomers MMA, METAC, NIPAm and GMA were as follows: MMA $6.0 \mathrm{ml}$, copper(I) bromide $(\mathrm{CuBr}) 0.14 \mathrm{~g}, 2,2^{\prime}$-dipyridyl (bipy) $0.3 \mathrm{~g}$, water/methanol $(30 \mathrm{ml}, 1 / 4 \mathrm{v} / \mathrm{v}) 45 \mathrm{~min}, 30^{\circ} \mathrm{C}$; METAC $6.9 \mathrm{~g}$, copper(I) bromide $(\mathrm{CuBr})$ 
$0.135 \mathrm{~g}, 2,2^{\prime}$-dipyridyl (bipy) $0.36 \mathrm{~g}$, water/methanol $(9 \mathrm{ml}, 2 / 7 \mathrm{v} / \mathrm{v})$ overnight, room temperature; NIPAm $1.5 \mathrm{~g}$, pentamethyldiethylenetriamine $165 \mu \mathrm{l}, \mathrm{CuBr}$ $48 \mathrm{mg}$, water/methanol $(30 \mathrm{ml}, 1 / 1 \mathrm{v} / \mathrm{v}) 30 \mathrm{~min}, 30^{\circ} \mathrm{C}$; GMA $6.0 \mathrm{ml}, \mathrm{CuBr}$ $63 \mathrm{mg}$, bipy $0.17 \mathrm{~g}$, water $/$ methanol $(30 \mathrm{ml}, 1 / 4 \mathrm{v} / \mathrm{v}) 50 \mathrm{~min}, 30^{\circ} \mathrm{C}$.

\section{Transfer of polymer@graphene 2D objects}

The $\mathrm{Cu}$ foil was etched away by placing the polymer-brush-grafted graphene/ $\mathrm{Cu}$ sample onto a $50 \mathrm{mg} \mathrm{ml}^{-1} \mathrm{FeCl}_{3} / \mathrm{H}_{2} \mathrm{O}$ solution surface for $2 \mathrm{~h}$. The freely floating $2 \mathrm{D}$ objects were retrieved using a glass slide and rinsed with de-ionized (DI) water several times to obtain the asymmetric functionalized graphene, which was transferred onto the target substrate for surface modification.

\section{Immobilization of biomolecules}

Poly(GMA) (PGMA)@G on PET was used to immobilize an oligonucleotide. A 20-mer, $5^{\prime}$-amine-modified oligonucleotide labeled with TET in the $3^{\prime}$ position was dissolved in $1 \times$ phosphate-buffered saline $(\mathrm{pH}=8)$ to prepare a $300 \mathrm{~nm}$ solution. Oligonucleotide patterns with $9 \times 9$ dot arrays were prepared by inkjet printing the $300 \mathrm{~nm}$ oligonucleotide solution onto the PGMA@G/PET surface. Then, the sample was placed in a sealed chamber with saturated $\mathrm{NaCl}$ solution overnight at room temperature. Unreacted oligonucleotides were removed by washing with $1 \times$ phosphate-buffered saline and DI water. Subsequently, the unreacted epoxy groups were blocked with ethanolamine, and the immobilized $5^{\prime}$-amine-modified oligonucleotide was hybridized with a TAMRA-labeled oligonucleotide.

\section{Fabrication of poly (MMA) pattern on $\mathrm{Cu}$ foil}

Femtosecond ( $\mathrm{fs}$ ) laser pulses were produced by a Ti:sapphire regenerative amplifier system (Spectra-Physics, Santa Clara, CA, USA). The laser pulses had a duration of $120 \mathrm{fs}$ and a repetition rate of $1 \mathrm{kHz}$ and were focused to a focal spot with a diameter of $\sim 2 \mu \mathrm{m}$ using a microscope objective $(\times 20$, $\mathrm{NA}=0.5$ ). The intensity of the laser pulse incident on the sample was controlled using a half-wave plate and a linear polarizer. The pulse energy used in the experiment was $\sim 10 \mu \mathrm{J}$. During the fabrication, the sample (poly MMA, PMMA, -grafted graphene on $\mathrm{Cu}$ foil) was mounted onto a computercontrolled three-axis translation stage with a moving resolution of $40 \mathrm{~nm}$. The moving speed was set to $\sim 50 \mu \mathrm{m} \mathrm{s}^{-1}$ for the square pattern.

\section{Instruments and characterization}

Optical and fluorescent images were recorded with a Nikon Eclipse 80i optical microscope (Nikon, Tokyo, Japan), $\lambda \mathrm{ex}=465-495 \mathrm{~nm}, \lambda \mathrm{obs}=515-555 \mathrm{~nm}$ for green fluorescence and $\lambda \mathrm{ex}=528-553 \mathrm{~nm}, \lambda \mathrm{obs}=577-632 \mathrm{~nm}$ for orange fluorescence. Atomic force microscopy (AFM) morphologies were measured by an XE-100 AFM (Park Systems, Suwon, South Korea) in non-contact mode under ambient conditions. Attenuated total reflection Fourier transfer infrared spectroscopy was performed using a PerkinElmer Spectrum 100 FI-TR spectrometer (PerkinElmer Inc., Waltham, MA, USA). Transmission electron microscopy (TEM) was performed using a JEM-2010 (JEOL, Tokyo, Japan) with an accelerating voltage of $120 \mathrm{kV}$. The transmittance spectra of the asprepared graphene thin films were collected using a UV-vis spectrometer (PerkinElmer Lambda 18). Raman spectra were collected using a Raman spectrometer (Renishaw microprobe RM 1000, Renishaw Plc., Gloucestershire, UK) with a 633-nm laser.

\section{RESULTS AND DISCUSSION}

In a typical experiment involving the preparation of polymer@graphene $2 \mathrm{D}$ objects, graphene grown by $\mathrm{CVD}$ on copper foils (graphene/Cu) was selected as the starting substrate. Graphene/Cu was first immersed in a DMF solution of ATRP macro-initiators, poly[2-(2-bromoisobutyryloxy) ethyl methacrylate-co-4-(1-pyrenyl) butyl methacrylate], in which the macro-initiator molecules were self-assembled onto graphene surfaces via $\pi-\pi$ interactions between pyrene side chains and the basal plane of graphene. ${ }^{22}$ As illustrated in Scheme 1 , initiator-modified graphene/Cu was placed in a polymerization solution to grow polymer brushes via SI-ATRP. ${ }^{23-26}$
Subsequently, the entire substrate was immersed in an iron (III) chloride aqueous solution to etch away the copper foil. After copious water rinsing, a freestanding and transparent polymer@graphene floating on the water/air interface could be clearly observed (Figure 1a). Importantly, the functionality of these polymer@ graphene2D objects can be tailored by simply changing the polymer brushes immobilized. As proof-of-concept, four different types of polymer brushes were synthesized, including hydrophobic PMMA, hydrophilic poly(METAC) (PMETAC), thermal responsive poly(NIPAm) (PNIPAm) and highly reactive PGMA (Scheme 1). The corresponding polymer@graphene 2D objects were denoted as PMMA@G, PMETAC@G, PNIPAm@G and PGMA@G, respectively.

Optical micrograph studies revealed that the transferred graphene surfaces were fully covered with polymer brushes (Supplementary Figure S1). Measured by AFMthe thicknesses of PMETAC@G, PMMA@G,PNIPAm@G and PGMA@G were 26, 75, 103 and $115 \mathrm{~nm}$, respectively (Figure 3a). Note that although SI-ATRP can control the thickness of the polymer brushes well by monitoring the polymerization kinetics, we did not vary the brush thickness in this work. All the 2D objects exhibited high transparency greater than $80 \%$ at $550 \mathrm{~nm}$ (Figure $3 \mathrm{~b}$ ). Note that the graphene sheets we used were 2-3 layers thick and absorbed $\sim 7 \%$ of the optical light. The polymer brushes contributed to the absorption of the other $5-12 \%$ of the optical light. In general, thicker polymer brushes exhibited stronger absorption.

Attenuated total reflection Fourier transfer infrared spectroscopy was used to identify the surface immobilization. The feature peak of the $\mathrm{C}=\mathrm{O}$ stretching of the macro-initiator at $1735 \mathrm{~cm}^{-1}$ (Figure 2a) indicated the successful attachment of the initiator on graphene (initiator-G). The absorption peak of $\mathrm{C}=\mathrm{O}$ of PMMA@G increased, which was ascribed to the successful grafting of PMMA. ${ }^{27}$ For PMETAC@G, peaks of $1486 \mathrm{~cm}^{-1}$ representing $\left(\mathrm{CH}_{3}\right)_{4} \mathrm{~N}^{+}$bending vibration and $1728 \mathrm{~cm}^{-1}$ representing carbonyl group stretching demonstrated the presence of PMETAC brushes. ${ }^{28}$ For PNIPAm@G, the characteristic $\mathrm{C}-\mathrm{N}$ stretching at $1650 \mathrm{~cm}^{-1}$ and $\mathrm{N}-\mathrm{H}$ bending at $1550 \mathrm{~cm}^{-1}$ also revealed the formation PNIPAm brushes on graphene. ${ }^{29}$ For PGMA@G, the $\mathrm{C}-\mathrm{O}$ stretching at $907 \mathrm{~cm}^{-1}$ provided evidence of the successful grafting, despite the $\mathrm{C}=\mathrm{O}$ stretching of the esters at $1738 \mathrm{~cm}^{-1}$. The Raman spectra showed that the D, G and 2D peaks of graphene appeared on all the quasi-2D polymers, and no obvious difference in the peak ratio was observed, suggesting that our synthesis did not lead to the destruction of the basal plane of graphene (Figure 2b). ${ }^{7,30}$

These polymer@graphene 2D objects could be transferred onto many different target substrates for characterization and future usage. For example, we used a TEM grid to acquire a freestanding quasi-2D polymer. The thin film that was stuck to one end of the TEM grid remained intact and curled after drying, indicating its excellent mechanical stability and flexibility (Figures $1 \mathrm{~b}$ and e). Apart from TEM grids, as-made polymer@graphene 2D objects could be readily transferred onto many other substrates including PTFE, $\mathrm{SiO}_{2}$, textile fabrics, quartz, gold and PET films (Figure 1 and Supplementary Figure S1).

Importantly, the size of the polymer@graphene2D objects can be scaled up simply by using larger graphene/Cu substrates. As proof-ofconcept, we fabricated a $7.5 \times 5 \mathrm{~cm}^{2}$ giant PMMA@G and transferred it onto PET (Figure 3c). Clear observation of the HK PolyU logo indicated the high transparency and uniformity of the as-made giant PMMA@G. The entire film was highly bendable without cracking because of the inherent softness of our quasi-2D polymers. 


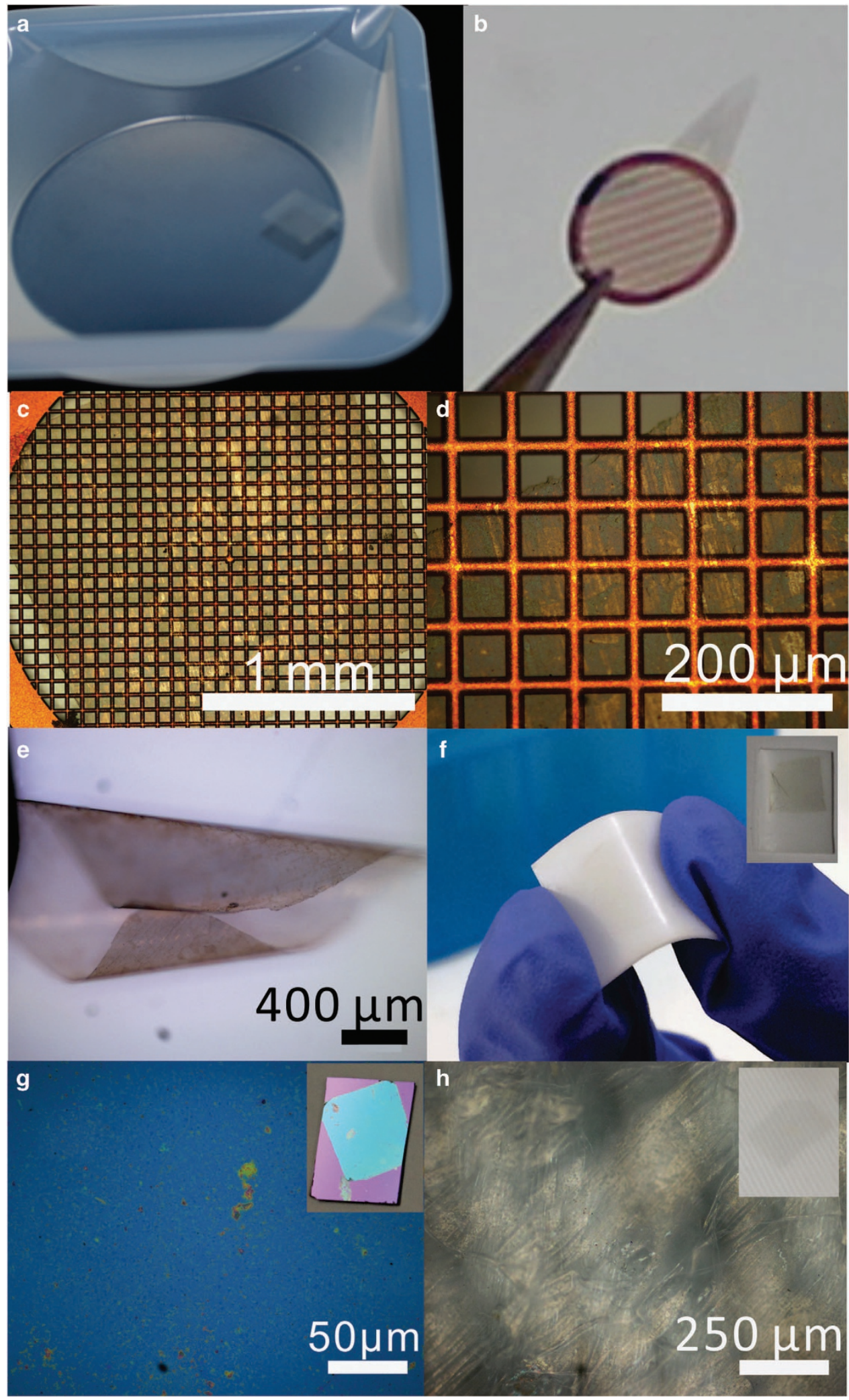

Figure 1 (a) Digital image of polymer@graphene floating on a water/air interface. (b) Photograph and (e) optical microscope image of a freestanding polymer@graphene with one end stuck on a TEM grid. (c) and (d) Microscope images of transferred PMMA@G film on copper grid with different magnifications. (f) Photograph of transferred PMMA@G on PTFE. Optical microscopic image of transferred PMMA@G on (g) $\mathrm{SiO}_{2}$ and (h) textile. 
a

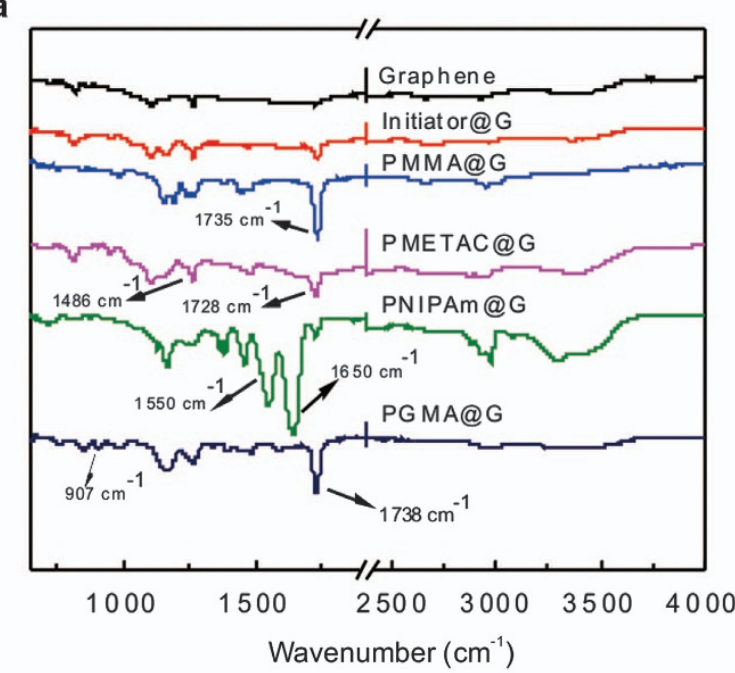

b

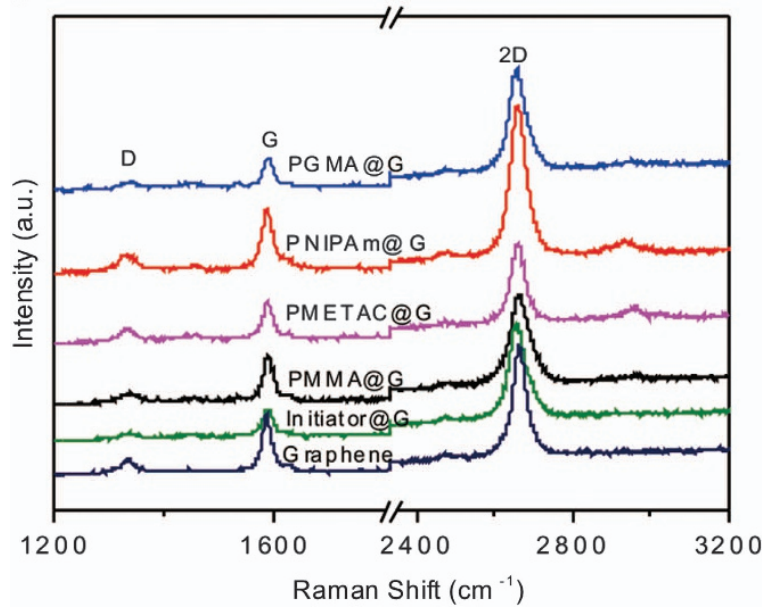

Figure 2 (a) Attenuated total reflection Fourier transfer infrared spectroscopy spectra and (b) Raman spectra of graphene, initiator@G, and four different types of polymer@graphene.

The polymer@graphene 2D objects were also durable in solventresistant tests. As a model test, PMMA@G transferred on PTFE and Si was dipped into different solvents, including $\mathrm{H}_{2} \mathrm{O}$, dimethyl sulfoxide and acetone, for more than 5 days. Photographs of these objects revealed that these thin films remained on the PTFE and Si after the solvent dipping tests (Supplementary Figure S2). AFM studies revealed that the film thickness and surface roughness only varied by $<10 \%$ when compared with the un-dipped samples, which might be attributed to the confirmation rearrangement of the polymer brushes in different solvents. These results illustrated that the non-covalent $\pi-\pi$ stacking interactions were strong enough to immobilize the polymer brushes against the solvation of the polymer chains, which is critical for these organic $2 \mathrm{D}$ materials because they are often exposed to good solvents of the polymer in different applications.

Currently, the degree of polymerization or the initiating efficiency of SI-ATRP is unknown. Determining the former has proven to be very challenging for all scientists working on polymer brushes. In theory, one can cleave off all the polymer brushes grown on the substrate and probe them using gel permeation chromatography.

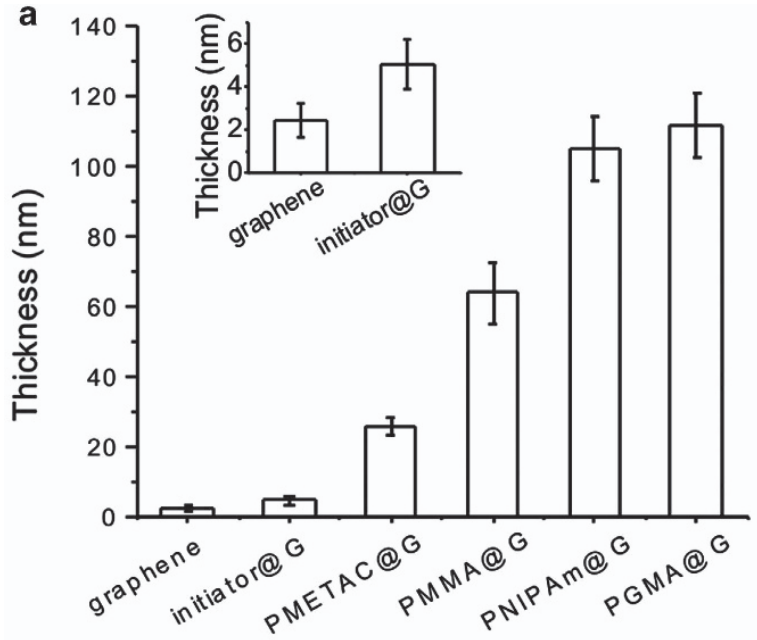

b
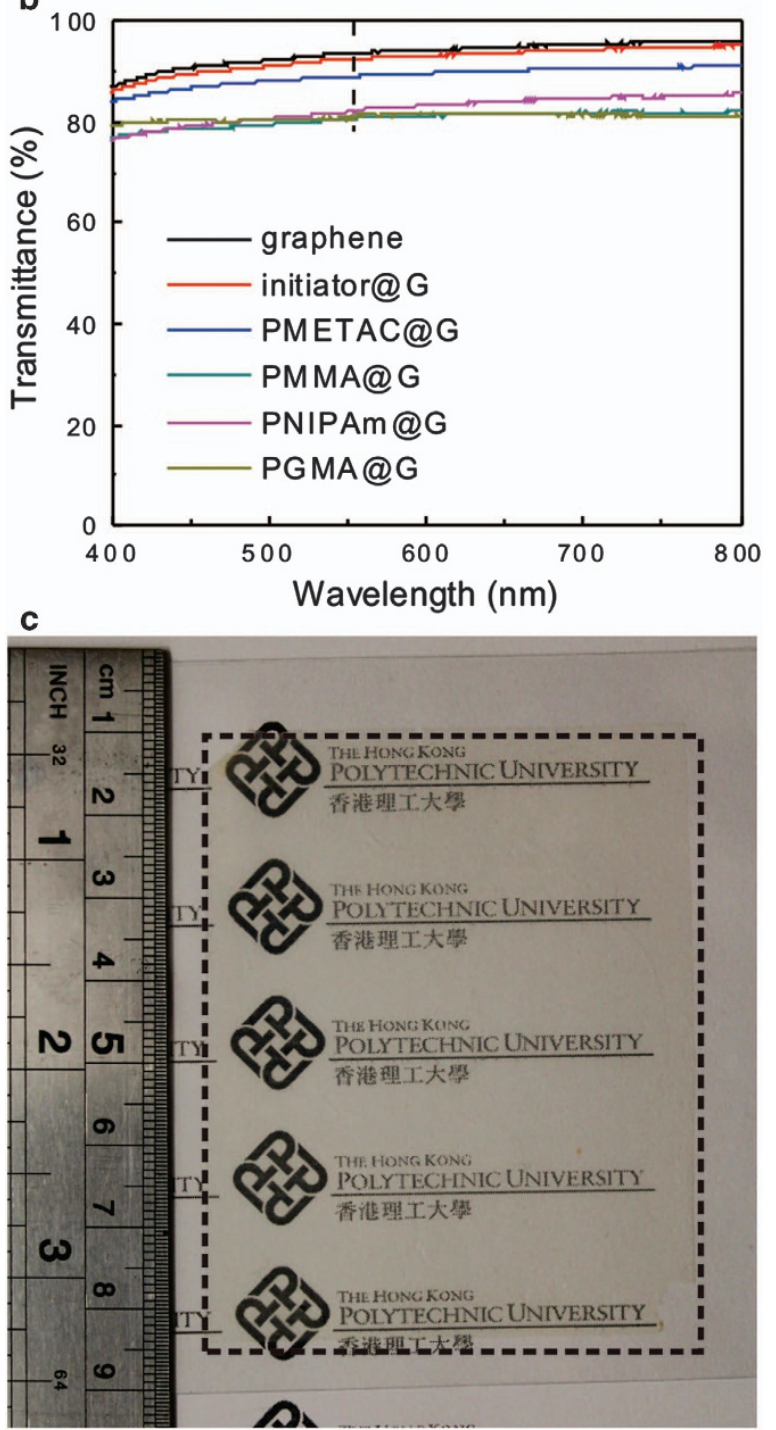

Figure 3 (a) Thickness and (b) transmittance of transferred graphene, initiator@G and different quasi-2D polymers (PMETAC@G, PMMA@G, PNIPAm@G and PGMA@G). (c) Photograph of a $7.5 \times 5 \mathrm{~cm}^{2}$ giant PMMA@G transferred on PET. 
However, typically, the amount of cleaved polymers is too low to acquire reliable results. Our polymer brushes are anchored firmly on graphene surfaces by strong $\pi-\pi$ interactions; therefore, it is practically difficult to cleave them off the surface. In terms of initiating efficiency, we are not able to detect the number of initiator sites functioning in SI-ATRP because of technical difficulties associated with the detection.

Because of the combinatorial advantages of low cost, high transparency, good solvent stability, abundant functions and feasibility to transfer, polymer@graphene 2D objects are particularly suitable for versatile surface modification and functionalization. Previous works using polymer brushes for surface modifications required direct chemical reaction on the substrates, which were difficult to clean and could contaminate other functional parts on the substrate. ${ }^{31}$ In addition, it is difficult to directly functionalize nonreactive polymer substrates, such as PTFE and PET, because of the lack of effective anchoring groups. These challenges can be readily overcome by transferring pre-made functional polymer@graphene 2D objects onto desirable substrates. As a proof-of-concept, we present two examples. The first example involves controlling the surface wettability. As demonstrated in Figure 4, after transferring PMMA@G onto $\mathrm{SiO}_{2}$, PET and PTFE, the contact angles of all substrates were $68-72^{\circ}$, which is a typical property of PMMA thin films. PNIPAm is a temperature-responsive polymer with a lower critical solution temperature of $\sim 32^{\circ} \mathrm{C}$. When transferring PNIPAm@G onto these substrates, all the surfaces became thermal responsive: when $\mathrm{T}<$ lower critical solution temperature, the contact angles were $\sim 60^{\circ}$. When $\mathrm{T}>$ lower critical solution temperature, the contact angles increased to $\sim 80^{\circ}$.

The second example involves the fabrication of DNA arrays on PET for sensor and diagnostic applications. PGMA is well known to react with amines and has been used to immobilize biomolecules, such as DNAs, proteins and enzymes. ${ }^{8,32}$ Here, we transferred PGMA@G onto PET. Then, arrays of $5^{\prime}$-amino-modified, $3^{\prime}$-TET-labeled singlestrand DNA oligonucleotides were inkjet-printed onto PGMA@G followed by overnight incubation, in which 3 -TET-labeled singlestrand DNA oligonucleotides was covalently bonded to the PGMA brushes through the ring-opening reaction of epoxy groups (Figure 5a). After rinsing away the physisorbed molecules, green fluorescence patterns were clearly observed under blue light excitation (Figures $5 \mathrm{~b}$ and c). 3'-TET-labeled single-strand DNA oligonucleotides was subsequently hybridized with its complementary strand by immersing the sample into a $100 \mathrm{~nm} 5^{\prime}$-TAMRA-labeled complementary DNA oligonucleotide solution for $7 \mathrm{~h}$ at $45^{\circ} \mathrm{C}$. After

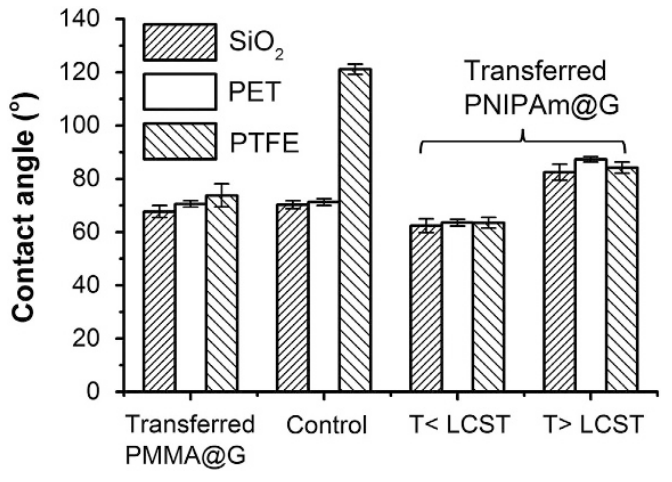

Figure 4 Contact angles of transferred polymer@graphene on different substrates for tuning the surface wettability.
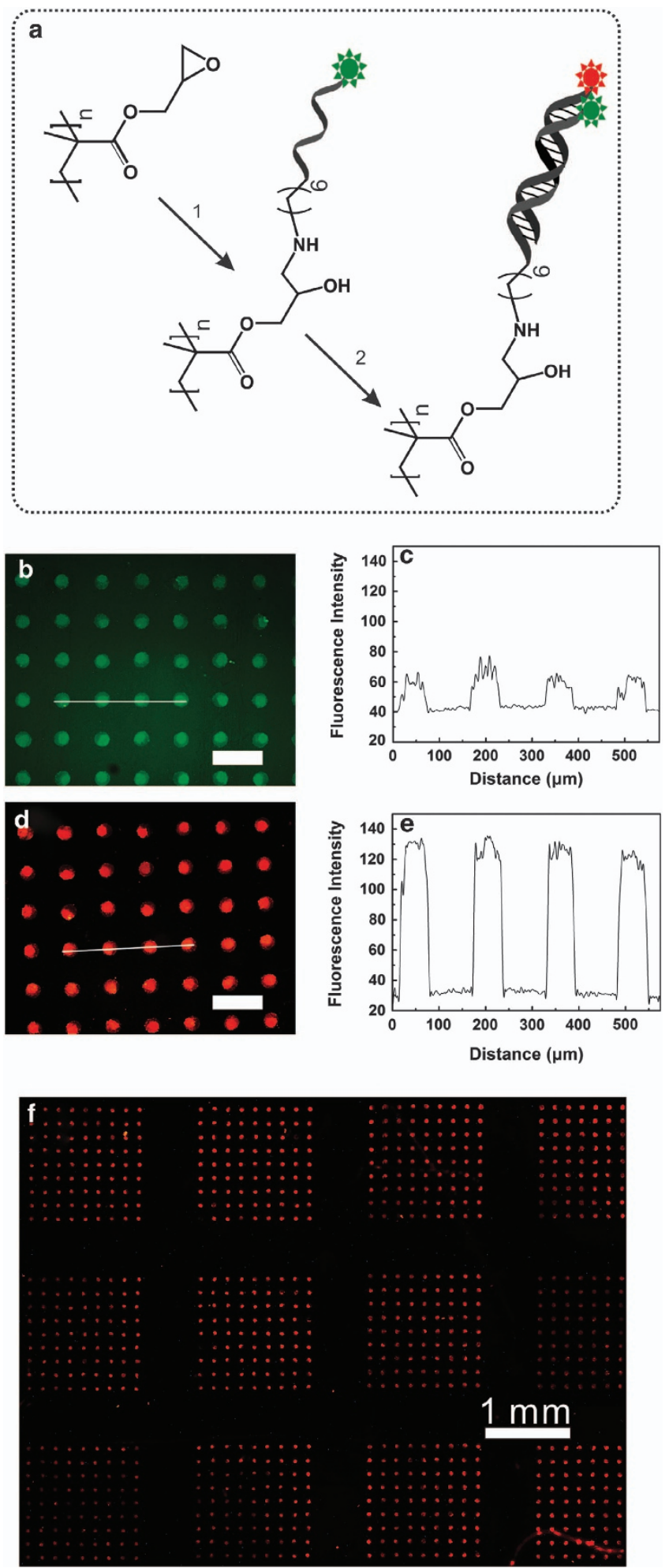

Figure 5 (a) Molecular structure illustration of the DNA binding and hybridization process. (b) Fluorescent microscope images and (c) corresponding cross-section analysis of fluorescence intensity of $5^{\prime}-\mathrm{NH} 2,3^{\prime}-\mathrm{TET}-$ modified ss-DNA bounded with PGMA@G. (d) Fluorescent microscope images. (e) The corresponding cross-section analysis of fluorescence intensity after hybridization with 5'-TAMRA-labeled complementary probes. The scale bars in the inserts are $200 \mu \mathrm{m}$. (f) A combined fluorescent microscope image of all the immobilized DNA arrays on PGMA@G transferred on PET. 

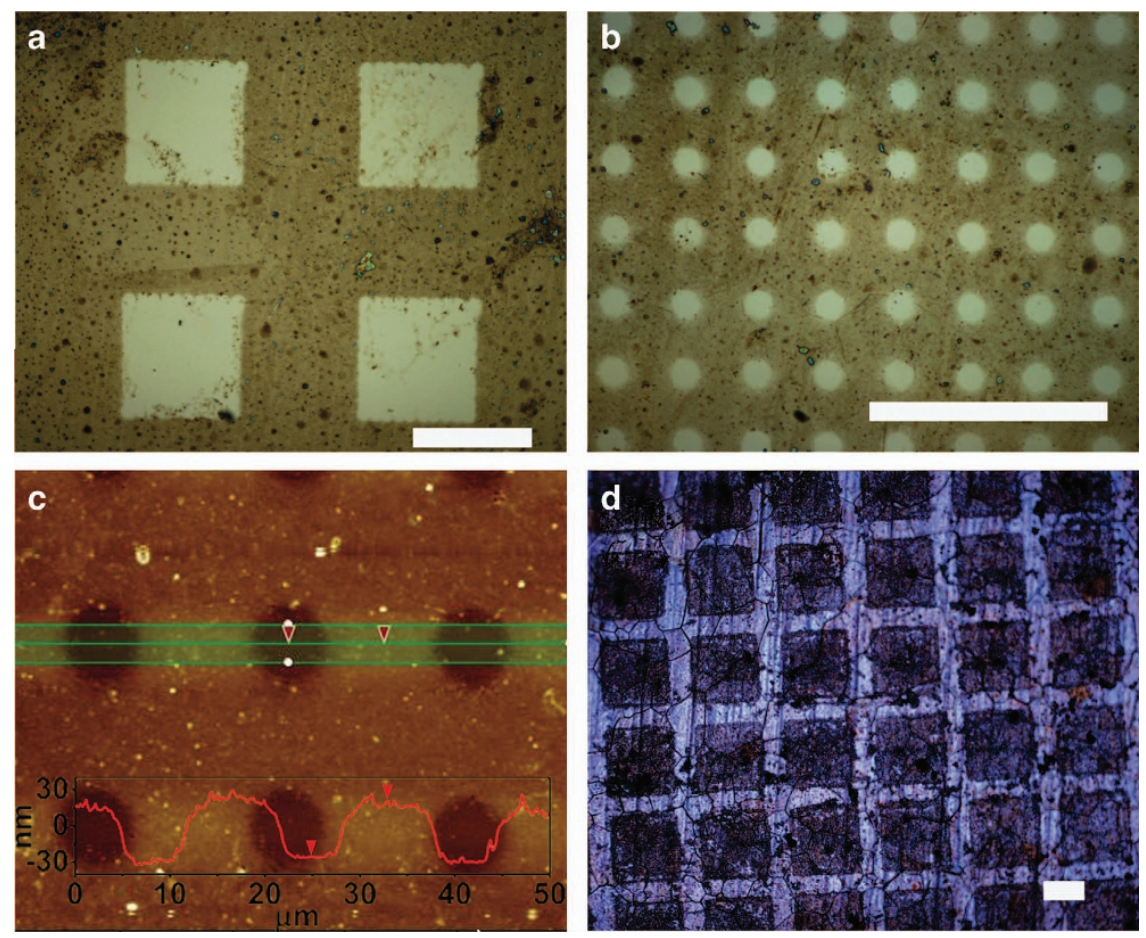

Figure 6 Patterned PMMA@G transferred on Si: (a,b) microscope images (scale bar $50 \mu \mathrm{m}$ ) and (c) AFM topography image. (d) Microscope image of patterned PNIPAm@G patterns transferred on $\mathrm{SiO}_{2}$.

hybridization and rinsing, orange fluorescence patterns were observed because of Forster resonance energy transfer, suggesting the successful hybridization of the two complementary oligonucleotide strands (Figures $5 \mathrm{~d}$ and e). Figure $5 \mathrm{f}$ presents a large-area fluorescent image of 12 as-made hybridized DNA arrays on PET.

Not only homogeneous films but also patterned quasi-2D polymers, which are mostly used as smart origami, can also be fabricated using polymer@graphene. As a proof-of-concept, we synthesized PMMA brushes on graphene/Cu. A femtosecond laser was then used to generate square or dot holes on the substrate. After etching the $\mathrm{Cu}$ foil and subsequent rinsing, patterned polymer@graphene objects were transferred onto Si. Optical microscopy revealed the as-made patterns with uniform $50-\mu \mathrm{m}$ squares and $5-\mu \mathrm{m}$ circles (Figures $6 \mathrm{a}$ and b). An AFM study also indicated that the patterns were regularly distributed on the substrate with a depth of $\sim 43 \mathrm{~nm}$ (Figure $6 \mathrm{c}$ ). In addition, we used microcontact printing to print the macro-initiator on graphene/Cu, followed by SI-ATRP of PNIPAm. Patterned PNIPAm brushes on graphene were generated. Again, after the etching, rinsing and transfer steps, the patterned quasi-2D polymers on $\mathrm{Si}$ could be observed (Figure $6 \mathrm{~d}$ ).

\section{CONCLUSIONS}

In conclusion, we have reported the preparation of a new type of organic 2D material, namely polymer@graphene 2D objects, using a low-cost and scalable chemistry. These freestanding 2D objects were shown to be lightweight, transparent, solvent stable, transferable to various substrates (e.g., PTFE, PET, Si, textiles), function-tailorable and patternable. We demonstrated their applications in the surface modification of non-reactive substrates (e.g., PTFE) to provide thermal-responsive surface wettability and in surface functionalization of PET to fabricate DNA arrays for detection purposes. We envision that with more advanced patterning techniques and materials design, numerous polymer@graphene 2D objects with different functionalities will be developed in the near future..$^{21,26,27,33-36}$

\section{CONFLICT OF INTEREST}

The authors declare no conflict of interest.

\section{ACKNOWLEDGEMENTS}

We acknowledge GRF of Hong Kong (PolyU 153041/14P) and The Hong Kong Polytechnic University (Project A-SA78) for financial support of this work.

1 Sakamoto, J., van Heijst, J., Lukin, O. \& Schlüter, A. D. Two-dimensional polymers: just a dream of synthetic chemists? Angew. Chem. Int. Edit. 48, 1030-1069 (2009).

2 Geim, A. K. \& Novoselov, K. S. The rise of graphene. Nat. Mater. 6, 183-191 (2007).

3 Bae, S., Kim, H., Lee, Y., Xu, X., Park, J. S., Zheng, Y., Balakrishnan, J., Lei, T., Kim, H. R., Song, Y. I., Kim, Y. J., Kim, K. S., Ozyilmaz, B., Ahn, J. H., Hong, B. H. \& lijima, S. Roll-to-roll production of 30-inch graphene films for transparent electrodes. Nat. Nanotechnol. 5, 574-578 (2010).

4 Chhowalla, M., Shin, H. S., Eda, G., Li, L. J., Loh, K. P. \& Zhang, H. The chemistry of two-dimensional layered transition metal dichalcogenide nanosheets. Nat. Chem. 5, 263-275 (2013).

5 Komsa, H. P. \& Krasheninnikov, A. V. Two-dimensional transition metal dichalcogenide alloys: stability and electronic properties. J. Phys. Chem. Lett. 3, 3652-3656 (2012).

6 Yang, D., Westreich, P. \& Frindt, R. F. Transition metal dichalcogenide/polymer nanocomposites. Nanostruct. Mater. 12, 467-470 (1999).

7 Baek, K., Yun, G., Kim, Y., Kim, D., Hota, R., Hwang, I., Xu, D., Ko, Y. H., Gu, G. H., Suh, J. H., Park, C. G., Sung, B. J. \& Kim, K. Free-standing, single-monomer-thick twodimensional polymers through covalent self-assembly in solution. J. Am. Chem. Soc. 135, 6523-6528 (2013).

8 Bhola, R., Payamyar, P., Murray, D. J., Kumar, B., Teator, A. J., Schmidt, M. U., Hammer, S. M., Saha, A., Sakamoto, J., Schlüter, A. D. \& King, B. T. A twodimensional polymer from the anthracene dimer and triptycene motifs. J. Am. Chem. Soc. 135, 14134-14141 (2013).

9 Colson, J. W. \& Dichtel, W. R. Rationally synthesized two-dimensional polymers. Nat. Chem. 5, 453-465 (2013).

10 Kissel, P., Erni, R., Schweizer, W. B., Rossell, M. D., King, B. T., Bauer, T., Götzinger, S., Schlüter, A. D. \& Sakamoto, J. A two-dimensional polymer prepared by organic synthesis. Nat. Chem. 4, 287-291 (2012). 
11 Edmondson, S. \& Huck, W. T. S. Quasi-2D polymer objects from patterned, crosslinked polymer brushes. Adv. Mater. 16, 1327-1331 (2004).

12 Kelby, T. S., Wang, M. \& Huck, W. T. S. Controlled folding of 2D Au-polymer brush composites into 3D microstructures. Adv. Funct. Mater. 21, 652-657 (2011)

13 Nardin, C., Winterhalter, M. \& Meier, W. Giant free-standing ABA triblock copolymer membranes. Langmuir 16, 7708-7712 (2000).

14 Salvatierra, R. V., Oliveira, M. M. \& Zarbin, A. J. G. One-pot synthesis and processing of transparent, conducting, and freestanding carbon nanotubes/polyaniline composite films. Chem. Mater. 22, 5222-5234 (2010).

15 Mattsson, J., Forrest, J. A. \& Börjesson, L. Quantifying glass transition behavior in ultrathin free-standing polymer films. Phys. Rev. E 62, 5187-5200 (2000).

16 Amin, I., Steenackers, M., Zhang, N., Beyer, A., Zhang, X., Pirzer, T., Hugel, T., Jordan, R. \& Gölzhäuser, A. Polymer carpets. Small. 6, 1623-1630 (2010).

17 Amin, I., Steenackers, M., Zhang, N., Schubel, R., Beyer, A., Gölzhäuser, A. \& Jordan, R. Patterned polymer carpets. Small. 7, 683-687 (2011).

18 Lee, C., Wei, X., Kysar, J. W. \& Hone, J. Measurement of the elastic properties and intrinsic strength of monolayer graphene. Science 321, 385-388 (2008).

19 Kim, J. Y., Kim, B. H., Hwang, J. O., Jeong, S. J., Shin, D. O., Mun, J. H., Choi, Y. J., Jin, H. M. \& Kim, S. O. Flexible and transferrable self-assembled nanopatterning on chemically modified graphene. Adv. Mater. 25, 1331-1335 (2013).

20 Seifert, M., Koch, A. H. R., Deubel, F., Simmet, T, Hess, L. H., Stutzmann, M., Jordan, R., Garrido, J. A. \& Sharp, I. D. Functional polymer brushes on hydrogenated graphene. Chem. Mater. 25, 466-470 (2013).

21 Steenackers, M., Gigler, A. M., Zhang, N., Deubel, F., Seifert, M., Hess, L. H. Lim, C. H., Loh, K. P., Garrido, J. A., Jordan, R., Stutzmann, M. \& Sharp, I. D. Polymer brushes on graphene. J. Am. Chem. Soc. 133, 10490-10498 (2011).

22 Gao, T. T., Wang, X., Yu, B., Wei, Q., Xia, Y. \& Zhou, F. Noncovalent microcontact printing for grafting patterned polymer brushes on graphene films. Langmuir $\mathbf{2 9}$ 1054-1060 (2013).

23 Liu, X. Q., Chang, H. X., Li, Y., Huck, W. T. S. \& Zheng, Z. J. Polyelectrolyte-bridged metal/cotton hierarchical structures for highly durable conductive yarns. ACS Appl. Mater. Interfaces 2, 529-535 (2010)

24 Ye, Q., Gao, T., Wan, F., Yu, B., Pei, X., Zhou, F. \& Xue, Q. Grafting poly(ionic liquid) brushes for anti-bacterial and anti-biofouling applications. J. Mater. Chem. 22, 13123-13131 (2012).

25 Ye, Q., Zhou, F. \& Liu, W. M. Bioinspired catecholic chemistry for surface modification. Chem. Soc. Rev. 40, 4244-4258 (2011).

26 Zhou, X. C., Wang, X. L., Shen, Y. D., Xie, Z. \& Zheng, Z. J. Fabrication of arbitrary three-dimensional polymer structures by rational control of the spacing between nanobrushes. Angew. Chem. Int. Edit. 50, 6506-6510 (2011).
27 Liu, X. Q., Li, Y. \& Zheng, Z. J. Programming nanostructures of polymer brushes by dip-pen nanodisplacement lithography (DNL). Nanoscale 2, 2614-2618 (2010).

28 Azzaroni, O., Moya, S. E., Brown, A. A., Zheng, Z., Donath, E. \& Huck, W. T. S. Polyelectrolyte Brushes as Ink Nanoreservoirs for Microcontact Printing of Ionic Species with Poly(dimethyl siloxane) Stamps. Adv. Funct. Mater. 16, 1037-1042 (2006).

29 Yang, D., Guo, G. Q., Hu, J. H., Wang, C. \& Jiang, D. L. Hydrothermal treatment to prepare hydroxyl group modified multi-walled carbon nanotubes. J. Mater. Chem. 18, 350-354 (2008).

30 Ferrari, A. C., Meyer, J. C., Scardaci, V., Casiraghi, C., Lazzeri, M., Mauri, F., Piscanec, S., Jiang, D., Novoselov, K. S., Roth, S. \& Geim, A. K. Raman spectrum of graphene and graphene layers. Phys. Rev. Lett. 97, 4 (2006).

31 Edmondson, S., Osborne, V. L. \& Huck, W. T. S. Polymer brushes via surface-initiated polymerizations. Chem. Soc. Rev. 33, 14-22 (2004).

32 Pirri, G., Chiari, M., Damin, F. \& Meo, A. Microarray glass slides coated with block copolymer brushes obtained by reversible addition chain-transfer polymerization. Anal. Chem. 78, 3118-3124 (2006).

33 Sankhe, A. Y., Booth, B. D., Wiker, N. J. \& Kilbey, S. M. Inkjet-printed monolayers as platforms for tethered polymers. Langmuir 21, 5332-5336 (2005).

34 Xie, Z., Shen, Y., Zhou, X., Yang, Y., Tang, Q., Miao, Q., Su, J., Wu, H. \& Zheng, Z. Polymer pen lithography using dual-elastomer tip arrays. Small. 8, 2664-2669 (2012).

35 Zhou, F., Zheng, Z. J., Yu, B., Liu, W. M. \& Huck, W. T. S. Multicomponent polymer brushes. J. Am. Chem. Soc. 128, 16253-16258 (2006).

36 Zhou, X. C., Liu, Z. L., Xie, Z., Liu, X. Q. \& Zheng, Z. J. High-resolution, large-area, serial fabrication of 3D polymer brush structures by parallel dip-pen nanodisplacement lithography. Small. 8, 3568-3572 (2012).

(c) (i) (2)(2) This work is licensed under a Creative Commons Attribution-NonCommercial-ShareAlike 3.0 Unported License. The images or other third party material in this article are included in the article's Creative Commons license, unless indicated otherwise in the credit line; if the material is not included under the Creative Commons license, users will need to obtain permission from the license holder to reproduce the material. To view a copy of this license, visit http://creativecommons.org/licenses/by-nc-sa/3.0/

Supplementary Information accompanies the paper on the NPG Asia Materials website (http://www.nature.com/am) 


\section{Transferable, transparent and functional polymer@graphene 2D objects}

Tingting Gao, Sze-Wing Ng, Xuqing Liu, Liyong Niu, Zhuang Xie, Ruisheng Guo, Chaojian Chen, Xuechang Zhou, Jun Ma, Wei Jin, Ying-San Chui, Wenjun Zhang, Feng Zhou and Zijian Zheng

NPG Asia Materials (2015) 7, e151; doi:10.1038/am.2014.119; published online 9 January 2015

Correction to: NPG Asia Materials (2014) 6, e130; doi:10.1038/ am.2014.79; published online 26 September 2014.

After online publication of this article, the authors noticed an error in the acknowledgement section.

The correct acknowledgement statement of this article should have read as below.
'We acknowledge GRF of Hong Kong (PolyU 153041/14P), The Hong Kong Polytechnic University (Project A-SA78) and NSFC $(21125316,21434009)$ for financial support of this work.'

The authors apologize for any inconvenience caused. 